\title{
REDUCTION OF PAPR IN MIMO-OFDM USING ADAPTIVE SLM AND PTS TECHNIQUE
}

\author{
CH. Nagaraju ${ }^{1}$, Anil Kumar Sharma ${ }^{2}$, M.V. Subramanyam ${ }^{3}$ \\ ${ }^{1}$ Research Scholar, Electronics \& Communication Engineering, Sunrise University-Alwar, Rajasthan \\ ${ }^{2}$ Professor \& Principal, Institute of Engineering \& Technology, Alwar, Rajasthan \\ ${ }^{3}$ Professor \& Principal, Santhiram Engineering College, Nandyal
}

Email : ${ }^{1}$ ch_nag2000@yahoo.com, ${ }^{2} a k s \_826 @ y a h o o . c o . i n,{ }^{3}$ mvsraj@yahoo.com

\section{Received : $06^{\text {th }}$ October 2017, Accepted $: 15^{\text {th }}$ November 2017, Published $: 31^{\text {st }}$ December 2017}

\begin{abstract}
This work will focus on performance comparison between two methods for combating the effects of PAPR: i.e. Selective Mapping (SLM) and Partial Transmit Sequence (PTS) method. These two methods are compared in terms of bandwidth efficiency and computation complexity with BER and PAPR reduction performance. This paper also describes the analysis and simulation of a modified SLM and PTS approach to decrease PAPR in MIMO-OFDM system. The basic principle of the coding technique known as STBC-OFDM is demonstrated through derivations and simulations. BER- analysis of MIMO-OFDM system using space-time block codes are simulated for Alamouti $2 \times 1$ system. The performance of the proposed system is better in terms of SNR as compared to systems with lower antenna configurations. In this work, the conjugate symbols were proposed, which have the same PA property. The proposed algorithm shows better PAPR performance and complexity reduction than the usual algorithms using the simulation results obtained.
\end{abstract}

\section{Keywords: MIMO-OFDM, PTS and SLM}

Technology, PAPR Performance.

\section{INTRODUCTION}

Life without a proper communication has become an intricate these days. Even a common man who doesn't have any knowledge of Communications enquires about the best communication service provider without any delays and thus leading to high data rate services. These services are always affected by various factors throughout the communication process, but so many novel approaches gave been established now and then in order to fulfil the requirements of users. Nowadays for high data rate services MIMO-OFDM system is well thought-out as one of the most exceptional methods. Mainly in wireless communications this system has hold its place firmly because of its advantages. Prior to this system a generic OFDM system is being explored for high speed data applications as OFDM offers power proficient signalling for a large number of subscribers on the same channel. MIMO-OFDM is used for numerous applications because of its high spectrum efficiency and the resistance to inter-symbol interference (ISI). PAPR issue is innate in MIMOOFDM system as it is developed in accordance with OFDM. The issue of PAPR is mainly dealt with a common problem in Communications i.e. signal distortion due to presence of independently modulated subcarriers and there arises a problem of Bit error rate (BER) [1]-[5]. In order to resolve BER an acquaintance of how different bit sequences are mapped. These days a number of modern sophisticated methods have been proposed in literature to reduce PAPR of OFDM Systems with a lot of ease [6]-[10]. Clipping is the easy and extensively used method to limit the PAPR below a threshold level; however it results in distortion and radiation (in-band and out-of-band). Another distinguished method to minimize PAPR is Block coding (the encoding of an input data into a codeword with low PAPR); consequently a decrease in data rate takes place. In this work an adaptive approach using selected mapping (SLM) and Partial transmit sequence (PTS) methods is proposed to minimize the PAPR without any signal distortion even though there is a chance of small increase in redundancy. Application of existing methods on each antenna is a clear-cut approach for reducing PAPR. Even though this approach is helpful for PAPR reduction without any difficulty, high complexity and large amount of side information (SI) are required [11]. Subsequently to solve this issue a contemporaneous algorithm is investigated, which selects the transmitted sequence with the minimum average (minaverage) PAPR over all transmit antennas. It lessens SI at the cost of a minor degradation of the PAPR performance. Nevertheless, the contemporaneous algorithm still requires numerous IFFT operations, which show high computational complexity. Here, a research on the property of space-time block coded (STBC) MIMOOFDM systems is carried out extensively. For unfussiness, a STBC MIMO-OFDM system that make use of Alamouti scheme was considered, and it is established that the conjugate symbols transmitted on two antennas have same property. As a result, novel contemporaneous algorithms with low complexity were proposed, which require only half of IFFT operations for usual contemporaneous algorithms. Furthermore, a new standard of minimum maximum (minmax) was proposed, in order to achieve better 
PAPR performance than the standard of minaverage in usual contemporaneous algorithms. And hence the work proposed in this paper may also be applicable to systems with multiple transmit antennas. The simulation results shown in this work are depicted in the form of graphical representations and tabular columns have been framed wherever necessary to show the comparative analysis based on various attributes. The paper has been noticeably organized in a chronological order of methods and results as various sections for an easy perception of the research work.

\section{DATA MODEL OF MIMO-OFDM AND PAPR REDUCTION ALGORITHMS}

\subsection{Mathematical model}

MIMO with OFDM system is parallel one as the regular OFDM it gives way to disadvantage of PAPR. There is a maximum probability of high PAPR when large number of sub carriers is present. High PAPR is not allowed through the high power amplifier which cuts the signal and causes distortion which intern creates enhancement of BER [11].

Now days, lot of techniques have cropped up to curb the PAPR of OFDM system. Block coding is another method which in low PAPR information encased in a code word. But this manifests in the loss in data rate. The techniques such as SLM and PTS act by cutting PAPR marginally incurring redundancy giving no room to signal distortion. MIMO-OFDM systems involve direct access for PAPR lowering by applying the usual algorithms individually on each transmit antenna. This is no doubt can lower PAPR but for drawback of needing high complexity and extensive side information (SI) [12]. To overcome this drawback concurrent algorithm is found which acts through distribution of minimum average PAPR on all transmit antennas.

The research on MIMO OFDM system along with STBC alamouti's scheme brings about transmission of conjugate symbols on two antennas having identical characteristics. In this work, we proposed a new concurrent algorithms having low complexity. These require only half the number of IFFT computations of existing algorithms.

Furthering the research activity to improve PAPR performance over the minimum average conventional concurrent algorithms, the minimum maximum criterion was brought about. In STBC-OFDM, the information symbols are coded across multiple antennas and time via the use of multiple consecutive OFDM symbols, whereas, SFBC symbols are coded across multiple antennas and multiple OFDM subcarriers. In this work, we present the performance analysis of various number of transmit and receive antennas for STBC-OFDM and SFBC-OFDM along with computational analysis and simulation plots [11]. However, STBC with real modulation would have better bandwidth efficiency performance than complex modulation. This is because STBC with real modulation require transmission of less data than STBC with complex modulation. On the other hand, STBC with two transmitting antennas always give better performance because they transmit more data. This would give the beneficiary capability to get the transmitted information. Besides, with bigger number of receive antennas, the same transmitted information would be gotten by more than one get receive antenna. A clear-cut Space-Time-Code for 2 TX, proposed by Alamouti [10]. The algorithm is as follows

1. Consider a transmission sequence, $\left\{s_{1}, s_{2}, s_{3}, \ldots, s_{n}\right\}$

2. Send $s_{1}, \quad s_{2}$ and, $s_{3} \ldots$.etc. in first, second and third time space respectively.

3. During the first time space, send $s_{1}, s_{2}$ . Subsequently $-s_{2}{ }^{*}$ and $s_{1}{ }^{*}, s_{3}$ and $s_{4},-s_{4}^{*}$ and $s_{3}{ }_{3}$, etc.

\subsection{2-Tx, 1-RxAlamouti STBC coding}
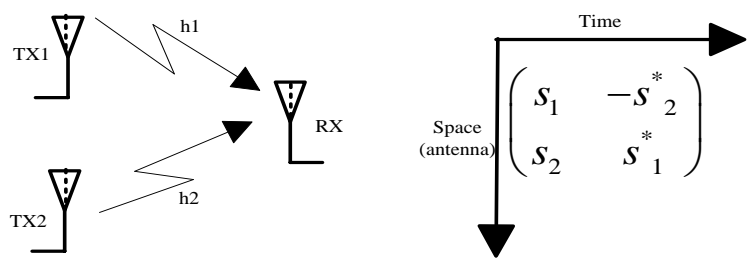

Fig. 1 2-Tx, 1-RxAlamouti STBC coding

Alamouti STBC- Receiver:

The signal received in first time space, is

$$
y_{1}=h_{1} s_{1}+h_{2} s_{2}+w_{1}=\left[\begin{array}{ll}
h_{1} & h_{2}
\end{array}\right]\left[\begin{array}{l}
s_{1} \\
s_{2}
\end{array}\right]+w_{1} \text {, }
$$

In the second time space, the signal received as,

$$
\begin{gathered}
y_{2}=-h_{1} s_{2}^{*}+h_{2} s_{1}^{*}+w_{2}=\left[\begin{array}{ll}
h_{1} & h_{2}
\end{array}\right]\left[\begin{array}{c}
-s_{2}^{*} \\
s_{1}^{*}
\end{array}\right]+2 x_{2} \\
\left.E\left\{\left[\begin{array}{l}
w_{1} \\
w_{2}^{*}
\end{array}\right]\left[\begin{array}{ll}
w_{1}^{*} & w_{2}
\end{array}\right]\right\}=\left[\begin{array}{cc}
\left|w_{1}\right|^{2} & 0 \\
0 & \left|w_{2}\right|^{2}
\end{array}\right] .3\right)
\end{gathered}
$$

matrix notation, the above

athematical statement can be defined as:

$$
\left[\begin{array}{l}
y_{1} \\
y_{2}^{*}
\end{array}\right]=\underbrace{\left[\begin{array}{cc}
h_{1} & h_{2} \\
h_{2}^{*} & -h_{1}^{*}
\end{array}\right]}\left[\begin{array}{l}
s_{1} \\
s_{2}
\end{array}\right]+\left[\begin{array}{l}
w_{1} \\
w_{2}^{*}
\end{array}\right]
$$


BER with Alamouti STBC

\section{In Rayleigh channel, the BER with alamouti STBC 2-transmit, 1- receive antenna is,}

$$
P_{e, S T B C}=P_{S T B C}^{2}\left[1+2\left(1-P_{S T B C}\right)\right],
$$

here

$$
P_{S T B C}=\frac{1}{2}-\frac{1}{2}\left(1+\frac{2}{\frac{E_{b}}{N_{0}}}\right)^{-\frac{1}{2}}
$$

Table 1. Attributes attained for MIMO-OFDM

\begin{tabular}{|c|c|}
\hline FFT Size & 64 \\
\hline umber of active subcarriers & 52 \\
\hline Band width & $16 \mathrm{MHz}$ \\
\hline Channel & Rayleigh \\
\hline $\begin{array}{c}\text { Sampling rate(fs) } \\
\text { ( } \Delta f \text { ) }\end{array}$ & $0.4 \mathrm{MHz}$ \\
\hline $\begin{array}{c}\text { Distance between adjacent } \\
\text { subcarrier }\end{array}$ & $312.5 \mathrm{kHz}$ \\
\hline $\begin{array}{c}\text { Symbol duration } \\
\text { Guard time }\end{array}$ & $3.2 \mu \mathrm{s}$ \\
\hline Total symbol duration & $0.8 \mu \mathrm{s}$ \\
\hline Moceive antenna & $4 \mu \mathrm{s}$ \\
\hline Transmit antenna & BPS and QPSK \\
\hline
\end{tabular}

Values of significant attributes have been tabulated in Table 1. In this section, Alamouti scheme and spacetime block codes encoding, decoding and BER performance analysis were covered and explained in detail. All simulation results were shown and explained in detail.

\subsection{Concurrent SLM}

Fig.2. shows the block diagram of concurrent SLM STBC Alamouti MIMO OFDM system.SLM can be used for any number of sub-carriers and for any signal constellation. It provides considerable gain with normal complexity. The side information is protected with Channel coding.
Hence one technique to lessen the in-phase addition is to change the phase before converting the frequency domain signal into time domain. It is common to apply SLM to each of the $M$ antennas in MIMO-OFDM individually, a methodology called individual SLM (ISLM).

Thus there is a requirement of applying IFFT and logarithmic value of bits of side information, as PAPR does not exceed the threshold and this discussion is simply given by the following equation

$$
\operatorname{Pr}\left(P A P R>P A P R_{0}\right)=1-\left(1-\left(1-\left(1-\mathrm{e}^{-P A P R_{0}}\right)^{N}\right)^{V}\right)^{M}
$$

To reduce signaling overhead, a concurrent SLM with illustrative block diagram as shown in Fig 2.10 was proposed. The subcarrier sequences from $N M$ transmit antennas are multiplied sub-carrier wise with the phase factor sequence $P^{(v)}=\left[P_{0}^{(v)}, P_{1}^{(v)}, \ldots, P_{N-1}^{(v)}\right] \ldots(1 \leq v \leq V)$ where each component is $P_{N}^{(v)} \in\{ \pm 1, \pm j\}$.

Among this the minimum average PAPR sequence over all transmit antennas is preferred. Subsequently, $\log _{2} V$ side information bits are required. In this case, the CCDF is written as

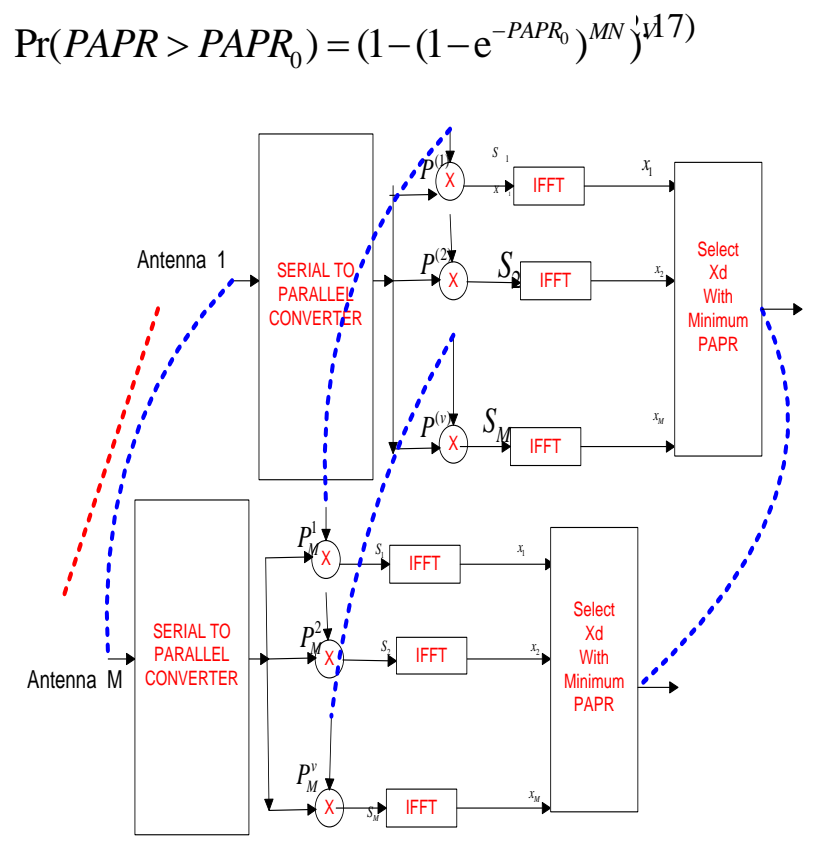

Fig. 2. Concurrent SLM

\subsection{Concurrent PTS}

One of the most generally used method for PAPR, PICR and PAR reduction by disjoint original data into sub-blocks is PTS. In conventional PTS algorithm, the input data block $X$ is divided into $D$ separate subblocks, as $X=\sum_{d=0}^{D-1} X^{(\mathrm{d})}$.These time-domain subblocks are rotated individually by the complex phase factor sequences 
$q^{(d)}=\left[b_{0}^{(d)}, b_{1}^{(d)}, \ldots, b_{N-1}^{(d)}\right] \ldots(1 \leq d \leq D)$ and

$b_{N}^{(d)} \in\left\{e^{\frac{j 2 \pi k}{W} a t k=0,1, W-1}\right\}$, where $W$ is the number of allowed phase factors.

Hence, the optimum set of phase factors need to be found by searching the $W^{D-1}$ sets of phase factors. Later the PAPR is evaluated for every subsequent sequence and then conveyed only with the minimum PAPR signal [11].

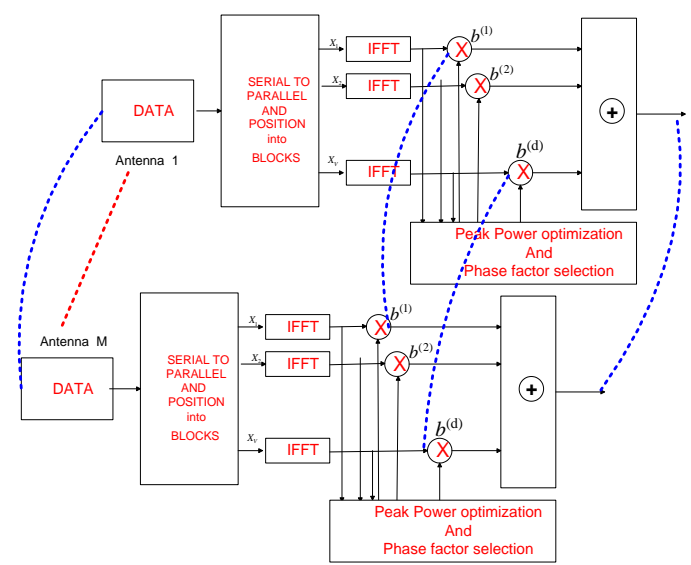

Fig. 3. Concurrent PTS

The block diagram represented in Fig.3. shows the working principle of Concurrent PTS, where the input data block is transformed into D parallel sequences and then the conventional PTS algorithm is applied for each sequence with the corresponding phase factor sequences being equal for all transmit antennas. This algorithm require $\log _{2} W^{D-1}$ bits of side information due to unique side information for all transmit antennas.

\section{PAPR REDUCTION ALGORITHMS IN STBC MIMO WITH OFDM USING PHASE FACTOR SEQUENCE MAPPING}

\subsection{Phase Factor Sequence Mapping}

Fig 4. shows Phase factor sequence mapping algorithm. During the first symbol period, the OFDM symbols $S_{1}$ and $S_{2}$ are transferred from antenna 1 and 2 respectively. Subsequently $-S_{2}{ }^{*}$ and $S_{1}{ }^{*}$ are transferred, where ()$^{*}$ designates Hermitian transpose.

PAPR reduction is performed on $S_{1}$ and $S_{2}$ to achieve two signals with low PAPR.

It is evident that $S_{i}$ and $\pm S_{i}(i=1,2)$ have the same PAPR property.Thus, the optimum phase factor sequences of $-S_{2}^{*}$ and $S_{1}^{*}$ can be achievedfrom $S_{1}$ and $S_{2}$, and the computation complexity reduced to half.
Fig. 4. Phase Factor Sequence Mapping Algorithm

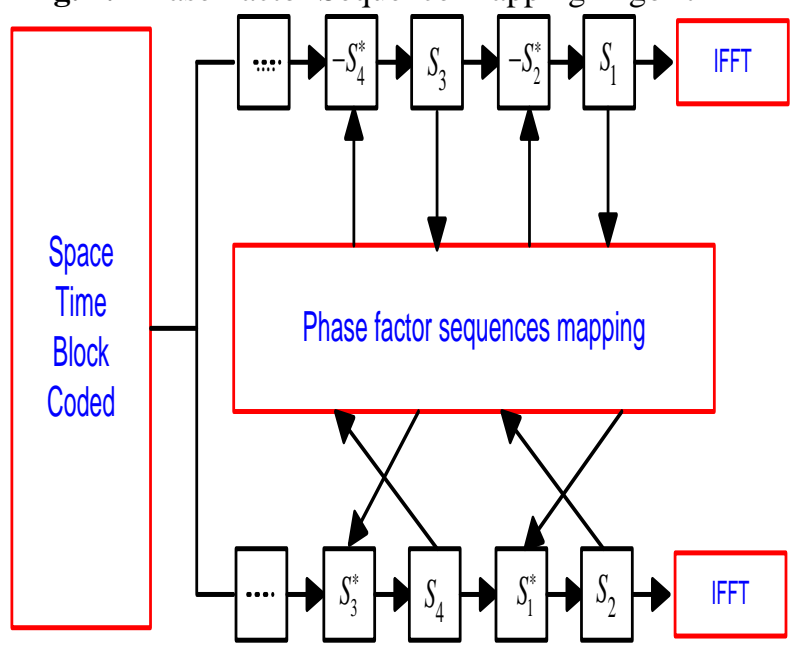

Table. 2 Phase factor sequence mapping

\begin{tabular}{|c|l|l|l|l|}
\hline Symbol & \multicolumn{4}{|c|}{ Phase factor sequence } \\
\hline$S_{i}$ & 1 & -1 & $j$ & $-j$ \\
\hline $\pm S_{i}^{*}$ & 1 & -1 & $-j$ & $j$ \\
\hline
\end{tabular}

\subsection{Modified concurrent SLM algorithm}

In this algorithm, initially the conventional SLM is applied on two symbols $S_{1}$ and $S_{2}$ along with the phase factor sequence mapping. Afterward the modified sequences $\widetilde{S_{1}}$ and $\widetilde{S_{2}}$ are achieved with low PAPR along with the optimum phase factor sequence $\widetilde{P}^{(v)}$. Subsequently we obtain the sequence with the same low PAPR. With reference to table 2 , it is clear that the phase factor sequences $\tilde{P}^{\prime(v)}$ are achieved from $\tilde{P}^{(v)}$ and the signals obtained by multiplying the phase factor sequences $\tilde{P}^{\prime(v)}$ are having similar PAPR property as $S_{i}$. Therefore, the computation complexity of modified concurrent SLM is reduced to half, compared to conventional SLM.

\section{Procedure for modified algorithm:}

1. Multiply the symbols $S_{1}$ and $S_{2}$ with the phase factor sequence $P^{(v)}$ and then determine PAPR $\left(S_{l}\right)$ and PAPR $\left(S_{2}\right)$

2. Determine PAPR maximum $\left\{\operatorname{PAPR}\left(S_{1}\right), \operatorname{PAPR}\left(S_{2}\right)\right\}$, and represent it as $P A P R(X)$

3. Repeat the above procedure for different phase factor sequences.

4. Select the minimum $\operatorname{PAPR}(X)$ along with the phase factor sequence $\widetilde{P}^{(v)}$

5. Next multiply the symbols $-S_{2}^{*}$ and $S_{1}^{*}$ with $\widetilde{P}^{\prime}(v)$, which is converted from $\widetilde{P}^{(v)}$ with reference to table. 2 . 
6.The above procedure is repeated till the end.

\subsection{Modified concurrent PTS algorithm}

The algorithm for modified concurrent PTS is as follows:

1. The symbols $S_{1}$ and $S_{2}$ from two transmit antennas are segregation into $D$ sub blocks sequentially, then multiplied with same combination of phase factor sequence $\left\{q^{(1)}, q^{(2)}, \ldots, q^{(d)}\right\}$. Calculate the PAPR of $S_{1}$ and $S_{2}$, and denote it as $\left\{\operatorname{PAPR}\left(S_{1}\right), \operatorname{PAPR}\left(S_{2}\right)\right\}$.

2. Find the maximum $\left\{\operatorname{PAPR}\left(S_{1}\right), \operatorname{PAPR}\left(S_{2}\right)\right\}$, and denote it as $\operatorname{PAPR}(X)$.

3 . Repeat the above procedure for different phase factor sequences.

4. Select minimum $P A P R(X)$, and the corresponding phase factor sequences $\left\{\tilde{q}^{(1)}, \tilde{q}^{(2)}, \ldots, \tilde{q}^{(d)}\right\}$ simultaneously.

5. The symbols $-S_{2}^{*}$ and $S_{1}^{*}$ are multiplied with $\left\{\tilde{q}^{\prime(1)}, \tilde{q}^{\prime(2)}, \ldots, \tilde{q}^{\prime(d)}\right\} \quad$ transformed from $\left\{\tilde{q}^{(1)}, \tilde{q}^{(2)}, \ldots, \tilde{q}^{(d)}\right\}$ according to Table 2.

6 . The above procedure is repeated till the end.

Only $\log _{2} W^{D-1}$ bits of side information are transmitted in Conventional PTS with $M D$ IFFT operations. Where $\mathrm{W}$ is the maximum number of granted phase factors. By using the proposed scheme, the number of IFFT operations is reduced to half compared with the Conventional PTS. We know that the complexity of one ordinary IFFT operation is $(N / 2) \log _{2} N$ multiplications and $N \log _{2} N$ additions.

\subsection{Analysis of Computation Complexity}

In conventional SLM, the number of IFFT operations and side information bits required are MV and $\log _{2} V$

, where as in proposed algorithm the computation complexity is reduced to half. Table 3 . describes the computation complexity for different versions of SLM. Similarly, the computation complexity for different versions of PTS is analyzed in table 4.

Table: 3 Computation complexity for different versions of SLM

\begin{tabular}{|c|c|c|}
\hline & Conventional SLM & Proposed SLM \\
\hline SI & $\log _{2} V$ & $\log _{2} V$ \\
\hline IFFT & $M V$ & $M V / 2$ \\
\hline Multiplication & $M V(N / 2) \log _{2} N$ & $M V(N / 4) \log _{2} N$ \\
\hline
\end{tabular}

\begin{tabular}{|l|l|l|}
\hline Addition & $M V N \log _{2} N$ & $M V(N / 2) \log _{2} N$ \\
\hline
\end{tabular}

Table: 4 Computation complexity for different versions of PTS

\begin{tabular}{|c|c|c|}
\hline & onventional PTS & Proposed PTS \\
\hline SI & $\log _{2} W^{D-1}$ & $\log _{2} W^{D-1}$ \\
\hline IFFT & $M D$ & $M D / 2$ \\
\hline Multiplication $M D(N / 2) \log _{2} M D(N / 4) \log _{2} N$ \\
\hline Addition & $M D N \log _{2} N M D(N / 2) \log _{2} N$ \\
\hline
\end{tabular}

\section{SIMULATION RESULTS AND ANALYSIS}

The simulation results have been obtained using MATLAB technical computing language. There are various toolboxes available in MATLAB but chiefly used toolboxes for this research work are Communications system toolbox and signal processing toolbox. Performance of the proposed method is depicted by simulation results and analysis. Figure No. 5 clearly shows the performance of diverse versions of SLM and PTS in terms of the CCDF. The simulation results obtained in this work, $10^{4}$ independent OFDM symbols are used and the number of subcarriers in each symbol is $\mathrm{N}=128$ with QAM modulation.

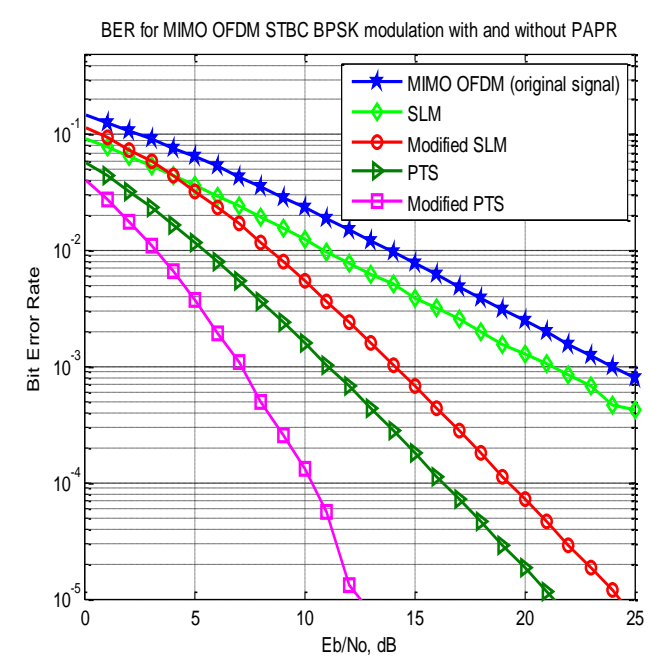

Fig. 5. PAPR reduction comparison analysis plot for STBC OFDM System

We assume two transmitting antennas (i.e. $M=2$ ). It is evident from Fig.5. that the proposed modified concurrent SLM algorithm outperforms the Conventional SLM at $\operatorname{Pr}\left(P A P R>P A P R_{0}\right)=10^{-1}$ i.eweachieve1.3dB PAPR reduction performance gain using the proposed algorithm compared to Conventional SLM. Similarly the performance of different versions of PTS algorithm is also demonstrated in Fig 5.When 
$\operatorname{Pr}\left(P A P R>P A P R_{0}\right)=10^{-1}, \quad$ the $P A P R_{0}$ of the original STBC OFDM is $10.1 \mathrm{~dB}$, Conventional PTS is $7.6 \mathrm{~dB}$ and the proposed modified concurrent PTS is $6.8 \mathrm{~dB}$, respectively i.e we achieve $0.8 \mathrm{~dB}$ PAPR reduction performance gain using the proposed modified concurrent PTS method compared with Conventional PTS.

Fig. 6. STBC OFDM System Performance

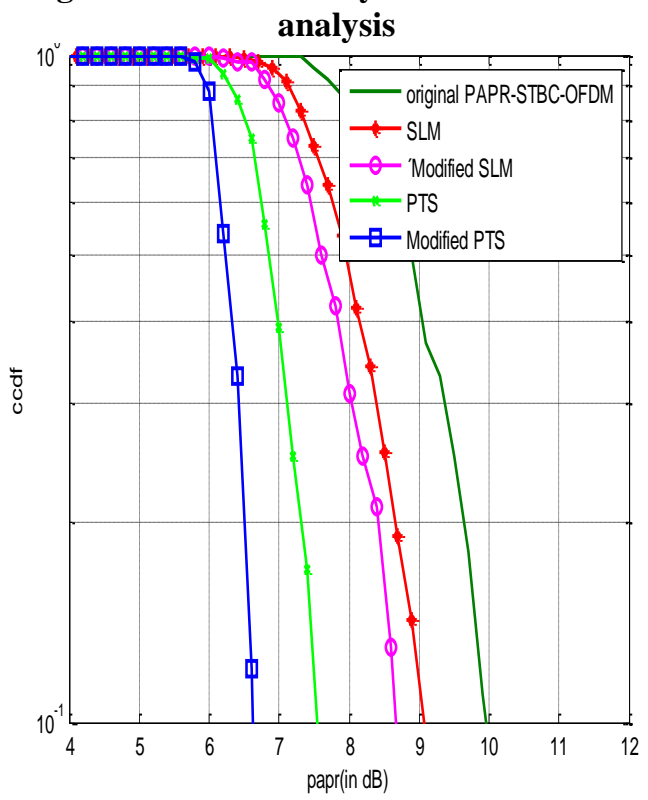

Numerous simulations have been carried out for the performance analysis of different STBC OFDM systems using versions of SLM/Modified SLM and versions of PTS/Modified PTS with number of transmit and receive antennas. In this computation work the channel coefficients are always assumed flat Rayleigh. From the plot shown in fig.6., it is evident that, the BER performance of proposed method is superior than the conventional methods.

Table-6. - Comparative table of SNR Values for different versions of SLM and PTS

\begin{tabular}{|c|c|c|c|c|c|}
\hline MULTIPLEXING & $\begin{array}{c}\text { Original } \\
\text { Signal }\end{array}$ & SLM & $\begin{array}{c}\text { Modified } \\
\text { SLM }\end{array}$ & PTS & $\begin{array}{c}\text { Modified } \\
\text { PTS }\end{array}$ \\
\hline $\begin{array}{c}\text { MIMO STBC OFDM } \\
\left(\text { BER 10 } 10^{-2}\right)\end{array}$ & $\sim 14 \mathrm{~dB}$ & $\sim 11 \mathrm{~dB}$ & $8.5 \mathrm{~dB}$ & $\sim 5.5 \mathrm{~dB}$ & $\sim 3.5 \mathrm{~dB}$ \\
\hline $\begin{array}{c}\text { MIMO STBC OFDM } \\
(\text { BER 10 }\end{array}$ & $\sim 24 \mathrm{~dB}$ & $-21.5 \mathrm{~dB}$ & $14.5 \mathrm{~dB}$ & $\sim 11 \mathrm{~dB}$ & $\sim 7 \mathrm{~dB}$ \\
\hline
\end{tabular}

A comparative table of SNR (Signal to Noise Ratio) values obtained for various methods is tabulated in Table 6. In communication systems SNR plays a prominent role to illustrate error probability with respect to the original signal present. From the comparative table it is apparent that for BER values of $\sim 10^{-3}$ and BPSK modulation the MIMO STBC OFDM shows in all cases the performance of modified

\section{Acknowledgements}

contemporaneous PTS is very high and it surpasses compared with the other schemes. The proposed modified SLM and PTS a based scheme provides better PAPR reduction capability as conventional SLM- or PTS-OFDM system. Mathematical as well as simulation results for BER performances confirm the outperformance of the proposed scheme over existing SI free schemes. Also, BER performance has been greatly improved. Though, the proposed technique reduces the PAPR, it is not efficient enough to maximize the spectral efficiency. Thus the proposed algorithm gives better minimum user capacity and sum capacity with low complexity compared to existing resource allocation algorithms for multi user SISO and MIMO-OFDM systems. The high throughput OFDM signal high PAPR problems are solved by the proposed methods of Modified Selected Mapping technique and Modified Partial Transmit Sequences techniques.

\section{CONCLUSION}

In this work, we consider phase factor sequence mapping with conjugate symbols and we prove that the symbols have the similar PA property. With this property, the computation complexity of proposed modified concurrent algorithms is reduced to half. The concept of PAPR in OFDM signals and PAPR reduction techniques like SLM and PTS have been investigated. The investigation has been proved in a right way using the plots obtained after simulation process. This paper also described modified SLM and PTS approach to reduce PAPR in MIMO-OFDM systems under Rayleigh fading channels. The basic principle of MIMO STBC-OFDM coding technique based on 'space and time' is demonstrated through derivations and simulations. BER- analysis of MIMOOFDM system using space-time block codes are simulated for Alamouti 2x1 system. The performance of the proposed system is better in terms of SNR as compared to systems with lower antenna configurations and this is shown in the paper with the help of a tabular column drawn for SNR values. In this work, the conjugate symbols were proposed, which have the same PA property. The proposed algorithm shows better PAPR performance and complexity reduction than the conventional algorithms.

The authors are thankful to Sunrise UniversityAlwar, Rajasthan and Annamacharya Institute of Technology \& Sciences, Rajampet, A.P. for providing research facilities.

\section{References}


1. Wulich D. "Definition of efficient PAPR in OFDM", IEEE Wireless Communications, Vol.9, No.9, pp.832-4, 2005.

2. Seung H H, Jae H L. "An Overview of peak-toaverage power ratio reduction", IEEE Wireless Communications, Vol.12, No.2, pp.56-65, 2005.

3. Sebastian caban, Christianmehlfauther, Robert langwieser, Arpad 1 scholtz, and markusrupp, "Matlab Interfaced MIMO Testbed", EURASIP Journal of applied signal processing, pp.1-13, June 2005.

4. Tarokh V, Jafarkhani H, Calderbank A R. "Space-time block codes from orthogonal designs", IEEE Transactions on Information Theory, Vol.45, No.5, pp.1456-1467, 1999.

5. Y. Ohwatari, B. H. Phu, Y. Ogawa, T. Nishimura, and T. Ohgane, "Reduction of the amount of Channel State Information Feedback in MIMOOFDM Eigen beam-Space Division Multiplexing Systems", Proc. IEEE VTC-Fall, pp. 1-5, Sep. 2006

6. W. C. Freitas Jr., A. L. F. de Almeida and F. R. P. Cavalcanti, "Interference Cancellation Receiver for Space Time Block Coded Systems over Frequency Selective Channels", Published by IEEE International Telecommunications Symposium, pp.555 - 559, Sep 2006

7. M.A.Mohammadu, M.Ardabilipour, B.Moussakhani and Z.Mobini, "Performance Comparison of RLS and LMS Channel Estimation Techniques with Optimum Training Sequences for MIMO-OFDM Systems", International conference on wireless and optical communications networks, pp.1-5, Mar 2008

8. Jun Ma, Philip Orlik, jinZhang,Geoffrey YE Li, "Pilot Matrix Design for Interim Channel Estimation in two Hopnmimo AF Relay Systems", IEEE International conference on communication(ICC), pp. 234-240, June 2009

9. hala $\mathrm{m}$ mahamoud, allam $\mathrm{s}$ mousa and rasheedsaleem, "channel estimation based on in comb type pilots arrangements for OFDM system over time varying channel", journal of networks, Vol.5,No. 7, pp.702-706,July 2010

10. N.Sreekanth and Dr.M.N.Giriprasad, "Signal to interference ratio (SIR) analysis and interference cancellation in MIMO OFDM systems with carrier frequency and timing offsets" IEEE Fourth International Conference on Communication and Computing (ARTCom), 2012.

11. Dae W L, Jong S N, Chi W L. (2005) 'A new SLM OFDM scheme with low complexity for PAPR reduction', IEEE Signal Processing Letters, Vol.12, No.2, pp.93-6.

12. Yung C T, Shang K D, Kuan C C. (2008) 'Turbo coded OFDM for reducing PAPR and error rates', IEEE Transactions on Wireless Communications, Vol.7, No.1, pp.84-9. ower ratio of multicarrier transmission schemes',
Electronics Letters, Vol.30. No.25, pp.20982099 